\title{
Mucormicosis, reporte de un caso y revisión de la literatura
}

Zavaleta Martínez Mariana*, Espinoza Acuña José Ricardo**, Ávalos Pavón Rafael ***

Alatorre Pérez Sergio****

\begin{tabular}{|c|c|}
\hline Resumen & Abstract \\
\hline $\begin{array}{l}\text { La mucormicosis es una enfermedad fúngica agresiva que causa in- } \\
\text { fecciones que potencialmente pueden ocasionar la muerte. Se ha } \\
\text { convertido en una enfermedad emergente debido, entre otros fac- } \\
\text { tores, al incremento de población inmunosuprimida, al uso de mejo- } \\
\text { res métodos diagnósticos y, posiblemente, al uso de antifúngicos de } \\
\text { amplio espectro. Se caracteriza por un desarrollo rápido de necrosis } \\
\text { tisular, resultado de la invasión vascular y la trombosis subsecuente. } \\
\text { Las formas de presentación son rino-órbito-cerebral (66\%), pulmonar } \\
\text { (I 6\%), cutánea, gastrointestinal ( I 0\%) o diseminada. Se presenta el } \\
\text { caso de una mujer de } 47 \text { años de edad, con mucormicosis, sin ante- } \\
\text { cedente de enfermedades crónicas, ni inmunocompromiso, a quien se } \\
\text { le realizó el diagnóstico intrahospitalario de diabetes mellitus tipo } 2 \text {. } \\
\text { LUX MÉDICA AÑO 14, NúMER0 42, SEPT-DIC 2019, PP 55-61. }\end{array}$ & $\begin{array}{l}\text { Mucormycosis is an aggressive fungal disease that causes infections } \\
\text { that can potentially cause death. It has become an emerging disease } \\
\text { due to the increase in immunosuppressed population, better diag- } \\
\text { nostic tests, and possibly to the use of broad-spectrum antifungals, } \\
\text { among other factors. It is characterized by the rapid development of } \\
\text { tissue necrosis, the result of vascular invasion and subsequent throm- } \\
\text { bosis. The forms of presentation are rhino-orbital-cerebral (66\%), pul- } \\
\text { monary (I6\%), cutaneous, gastrointestinal (10\%), or disseminated. } \\
\text { We present the case of a } 47 \text {-year-old woman with mucormycosis, } \\
\text { without a history of chronic diseases, or immunocompromise, who } \\
\text { had an in-hospital diagnosis of type } 2 \text { diabetes mellitus. LUX MÉDICA } \\
\text { AÑ 14, NÚMERO 42, SEPT-DIC 2019, PP 55-61. }\end{array}$ \\
\hline $\begin{array}{r}\text { Palabras clave: mucormicosis, rhizopus, diabetes } \\
\text { mellitus tipo } 2 .\end{array}$ & $\begin{array}{l}\text { Keywords: mucormycosis, rhizopus, type } 2 \text { diabetes } \\
\text { mellitus }\end{array}$ \\
\hline
\end{tabular}

\section{Introducción}

La mucormicosis se manifiesta por una variedad de diferentes síndromes clínicos que afecta, generalmente, a pacientes inmunocomprometidos; su manifestación más común se caracteriza por presentaciones infecciosas severas rino-orbitales, cerebrales y pulmonares, constituyendo los síndromes más comunes causados por estos hongos. ${ }^{1}$

Los géneros en el orden mucorales causan la mayoría de las infecciones humanas, éstos se caracterizan por su rápido crecimiento y liberan grandes cantidades de esporas que pueden dispersarse en el aire. Se distinguen, morfológicamente por sus anchas hifas

\footnotetext{
Médico Internista adscrito al Centenario Hospital Miguel Hidalgo Aguascalientes.

** Residente de primer año de Medicina Interna del Centenario Hospital Miguel Hidalgo

*** Residente de segundo año de Medicina Interna del Centenario Hospital Miguel Hidalgo

**** Médico Cirujano Maxilo-facial del Centenario Hospital Miguel Hidalgo

Fecha de recibido 1 de marzo 2019

Fecha de aceptación 1 de agosto 2019

Correspondencia: Dra Mariana Zavaleta Martínez. Servicio de Medicina Interna del Centenario Hospital Miguel Hidalgo. Avenida Gómez Morín S/N, Colonia La Estación, Alameda, Código postal 20259. Aguascalientes, Ags., México. Teléfono (449) 99467 20. Correo electrónico: zavaletaalfil@gmail.com
} 
(5 a 15 micrones de diámetro), con ramificaciones irregulares y con septos irregulares o en su mayoría por la ausencia de éstos. ${ }^{2}$

Al encontrarse libremente en el aire, le confina la característica de ser un alto contaminante de cultivos en laboratorio. Se han considerado múltiples factores de riesgo. Los pacientes que se ven afectados por éstos tienden a presentar disminución de la inmunidad, lo cual predispone tanto a la infección como en la severidad de la presentación clínica y respuesta al tratamiento. Se han realizado múltiples reportes de caso donde se ha observado alta incidencia en pacientes con diabetes mellitus, hasta en el $36 \%$ de los casos, seguido de las neoplasias hematológicas con neutropenias profundas hasta en un $17 \% .^{2,3}$ Otras personas con alto riesgo son aquellos pacientes con tratamiento crónico o a dosis altas de glucocorticoides, sometidos a trasplante de células hematopoyéticas o de órganos sólidos, ${ }^{4}$ pacientes con SIDA, o usuarios de drogas inyectables, incluso pacientes con antecedente de traumatismos, o quemaduras mayores al 33\% de la superficie corporal, en los que existe un daño extenso de tejidos blandos, ${ }^{5,6}$ y desnutrición proteico calórica severa.

La incidencia de la mucormicosis es difícil de estimar, ya que no es una enfermedad notificable y el riesgo varía ampliamente en diferentes poblaciones; se han notificado casos de mucormicosis en donde la piel ha sido el sitio de infección más común (en 57\%), seguido del tracto gastrointestinal (15\%). Los portales de entrada incluían cirugía, catéteres (especialmente catéteres intravasculares) y cinta adhesiva. Los brotes y los grupos se han asociado con vendajes adhesivos, depresores con lengüetas de madera, construcción de edificios adyacentes y ropa de cama de hospital.7,8

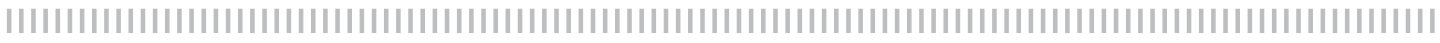

\section{Presentación del caso clínico}

Paciente femenino de 47 años de edad, originaria y residente de Aguascalientes, Aguascalientes, divorciada, escolaridad primaria, dedicada al hogar. Sin antecedentes heredofamiliares de neoplasias, enfermedades crónicas, tuberculosis u otras, no tiene descendencia. No tiene toxicomanías ni conductas de riesgo. Independiente para realizar las actividades de la vida diaria. Acude a valoración por iniciar cuadro clínico con aumento del volumen facial en región maxilar izquierda acompañado de dolor tipo punzante de intensidad subjetiva $6 / 10$ de la escala visual análoga del dolor, por lo que acude a clínica privada donde hacen diagnóstico de absceso dental, realizan drenaje y es dada de alta con amoxicilina e ibuprofeno, con lo que obtiene mejora parcial. Acude 20 días después de la extracción dentaria a valoración por el servicio de cirugía maxilofacial, donde se le indica tratamiento con clindamicina y se egresa con cita 10 días después para revaloración.

Es valorada por cirugía maxilofacial y se diagnostica absceso del espacio canino asociado a celulitis periorbitaria, inician tratamiento con ceftriaxona y continúan con clindamicina; se solicita tomografía donde se observa pérdida de la continuidad cor- 
tical del piso y pared lateral media del antro maxilar. Durante su estancia hospitalaria se documenta hiperglucemia de $449 \mathrm{mg} / \mathrm{dl}$, leucocitos de 19,034 con neutrófilos de 15,090 por lo que se inicia tratamiento con insulina glargina y se programa para drenaje del absceso nasogeniano. Se documenta sinusitis maxilo-etmoidal, se toma muestra para análisis histopatológico donde se tiene el hallazgo de mucormucosis, por lo que se inicia tratamiento con anfotericina $B$ a dosis de $1.5 \mathrm{mg} / \mathrm{kg}$ y se procede a etmoidectomía anterior y enucleación de globo ocular izquierdo. A los 5 días del postquirúrgico se efectúa endoscopía nasal izquierda con endoscopio flexible y se observa cornete medio e inferior, septum, piso nasal, seno maxilar de características normales y etmoides anterior en proceso de cicatrización. La paciente recibió un total de ocho semanas de tratamiento con anfotericina B. Durante su estancia intrahospitalaria se vigiló estrechamente la posibilidad de desarrollo de hipokalemia o hipomagnesemia, efectos secundarios esperados en personas que reciben tratamiento con anfotericina, presentándose leve hipokalemia que se manejó con aporte en soluciones salinas y dieta con mayor aporte de potasio. El seguimiento a un año después de la intervención quirúrgica ha sido satisfactorio y con control adecuado de sus cifras de glucosa.

\section{Discusión}

La mucormicosis se caracteriza por infarto y necrosis de los tejidos blandos, de evolución rápida, aunque hay reportes de casos con una evolución indolente. ${ }^{9} \mathrm{La}$ presentación clínica más frecuente es la infección de la mucormicosis rino-orbitalcerebral. Se presenta como una sinusitis aguda con fiebre, congestión nasal, secreción nasal purulenta y cefalea que tiende a involucrar estructuras contiguas, como el paladar, la órbita y el cerebro. Puede haber cuadros aparatosos como el reportado por Castrejón-Pérez y cols. donde la lesión más frecuente es una lesión ulcerosa con bordes necróticos, que al presentarse habitualmente ya se tienen daños severos a la órbita. ${ }^{10}$

A nivel de paladar, se observa una escara negra resultado de la necrosis tisular, acompañada de edema, eritema y cianosis. Los signos de afectación orbital incluyen edema periorbital, proptosis y ceguera, por afectación a las diferentes ramas de los nervios craneales. ${ }^{11}$ Se presenta un caso clínico de una mujer de 50 años de edad que tiene un cuadro similar al de la paciente que interesa, quien acudió a consulta por edema de hemicara derecha de 4 meses de evolución, quien se había sometido a extracción dental por dolor molar, sin mejoría del cuadro clínico y sin lograr adecuada cicatrización en el hueco de alveolo dentario, aunado a historia de mal control de glucosa, y presentaba glucosa en ayuno persistente en $154 \mathrm{mg} / \mathrm{dl}$ pese a tratamiento con hipoglucemiantes orales; en dicha paciente se realiza biopsia de la lesión dentaria y se hace diagnóstico histopatológico de mucormicosis. ${ }^{12}$

La necrosis o escaras en la cavidad nasal o paladar, la parálisis de nervios craneales, oftalmoplejía y pérdida de la visión son datos de progresión de la enfermedad. Las complicaciones intracraneales incluyen abscesos subdurales principalmente.

En el caso de la paciente del presente estudio no se presentó afección pulmonar, ya que de haberse presentado se esperaría una neumonía atípica con infarto y necrosis, y síntomas como tos, dolor pleurítico y disnea; con diseminación a estructuras contiguas, como el mediastino y el corazón o diseminación hematógena a otros órga- 
nos siendo la principal sintomatología fiebre y hemoptisis, ésta última por invasión vascular por mucor. ${ }^{13}$

Cuando la mucormicosis tiene afección gastrointestinal, suele asentarse principalmente en estómago y se manifiesta como dolor abdominal intenso, sangrado de tubo digestivo por úlceras necróticas que pueden conducir a perforación y peritonitis; ${ }^{14}$ situación tampoco presentada en la paciente en cuestión.

Mucormicosis cutánea: su forma de presentación generalmente es un área de celulitis similar a ectima que puede evolucionar a necrosis que generalmente se mantiene en tejidos blandos, y se adquiere por inoculación directa de esporas.

El mecanismo de infección del sistema nervioso central es por continuidad, generalmente de afección de senos paranasales o por fungemias las manifestaciones son tan variadas como el territorio cerebral afectado presentado desde letargo hasta déficits neurológicos más focalizados. ${ }^{15}$

El diagnóstico definitivo es con la identificación de organismos en el tejido mediante histopatología con confirmación de cultivo. Las muestras deben ser inspeccionadas para detectar hifas amplias, no separadas, con ramificación en ángulo recto, usando tinciones de calcoflúor blanco y metenamina de plata. La presencia de las hifas características en una muestra clínica proporciona un diagnóstico presuntivo que debería impulsar una evaluación adicional. Sin embargo, la ausencia de hifas no debe disuadir a los médicos del diagnóstico de mucormicosis cuando el cuadro clínico es altamente sugestivo. El examen histopatológico permite diferenciar morfológicamente las hifas de aspergillus y mucorales, lo cual es importante para tomar decisión del tratamiento a iniciar. Las pruebas de suero, como el ensayo de 1,3-beta-D-glucano y el ensayo de galactomanano de Aspergillus, se utilizan con mayor frecuencia en pacientes sospechosos de tener una infección micótica invasiva, que al reportarse como positiva, descartaría la infección por mucormicosis.

Aunque existen pocos estudios de investigación con pocos reportes de caso, se ha demostrado que técnicas moleculares como la reacción en cadena de la polimerasa (PCR) en muestras histológicas puede tener una alta sensibilidad y especificidad para el diagnóstico de mucormicosis y su tipificación; sin embargo, no se encuentra disponible en este medio. ${ }^{16}$

En caso de afección pulmonar, el diagnóstico es difícil porque la presentación no difiere de la neumonía debida a otros mohos angioinvasivos. Las radiografías de tórax o tomografías computarizadas pueden mostrar consolidación focal, masas, derrames pleurales o nódulos múltiples. Un signo de halo (atenuación de vidrio esmerilado que rodea un nódulo) es característico de los hongos angioinvasivos, pero también se ha informado de un signo de halo invertido, un área focal de la atenuación de vidrio esmerilado rodeada por un anillo de consolidación. ${ }^{17}$ Las muestras de esputo o de lavado broncoalveolar (LBA) pueden mostrar las hifas anormales y no sembradas, que suelen ser el primer indicador de mucormicosis; sin embargo, estas pruebas tienen un rendimiento bajo para el diagnóstico. ${ }^{17}$

El tratamiento debe de individualizarse siempre tratando de actuar sobre los factores de riesgo predisponentes, como en el caso de la paciente en quien se inició tratamiento con insulina glargina por diagnóstico de diabetes mellitus tipo 2 debutante. Además de un manejo dual con el debridamiento de tejido necrótico y la terapia antifúngica y las afecciones secundarias que se vayan detectando. ${ }^{18}$

La anfotericina B intravenosa (IV) (formulación lipídica) es el fármaco de elección para la terapia inicial. ${ }^{19}$ Los pacientes que reciben anfotericina $B$ liposomal como terapia primaria tienen una respuesta superior respecto a otras formas de presentación como la anfotericina B deoxicolato, 
teniendo una respuesta al tratamiento, con una supervivencia $67 \%$ vs $39 \%$ respectivamente. Las ventajas de las formulaciones lipídicas de la anfotericina $\mathrm{B}$ pueden resultar de la mejor penetración cerebral, disminución de la carga fúngica y efectos inmunomoduladores, además de tener menos efectos nefrotóxicos.

Posaconazol o isavuconazol se emplea como terapia en descenso para los pacientes que han respondido a la anfotericina $B$. También se puede utilizar como terapia de rescate para pacientes que no responden o no toleran la anfotericina $B$; para la terapia de rescate, la decisión de usarlos por vía oral o IV depende de la gravedad del paciente.

Para tener un tratamiento exitoso se requiere diagnóstico temprano, tratar los factores de riesgo, de ser posible, disminuir la inmunosupresión y la administración de antifúngicos. Se ha encontrado que un retraso en el tratamiento mayor a 6 días después del diagnóstico se asocia con el doble de mortalidad a las 12 semanas. El inicio temprano de la terapia antifúngica dentro de la primera semana, disminuye la mortalidad hasta en un $50 \% .20$

En cuanto a tratamiento debido a sus efectos menos nefrotóxicos se prefiere la administración de anfotericina B liposomal a una dosis de $5 \mathrm{mg} / \mathrm{kg}$ por día; sin embargo, no existen estudios que evalúen la dosis precisa. Para los pacientes que han respondido a una formulación lipídica de anfotericina $B$, se puede usar posaconazol o isavuconazol para la terapia de reducción oral. La terapia a menudo se extiende durante meses, y algunos pacientes permanecen en terapia de por vida si la inmunosupresión no se puede corregir.

Se recomienda el tratamiento de manera inicial con posaconazol o isavuconazol para pacientes que no responden o no pueden tolerar la anfotericina B. ${ }^{21}$ Como lo reportado en el trabajo de Ilharco $M$ y cols., ${ }^{22}$ donde pacientes que tienen polifarmacia o en aquellos que se presentó profunda hipomagnesemia e hipokalemia. ${ }^{21}$

\section{Conclusiones}

La mucormicosis puede ser la primera manifestación de la diabetes mellitus, y no sólo una manifestación de complicaciones por inmunodeficiencia primaria o secundaria. El diagnóstico temprano puede limitar la enucleación en caso de mucormicosis rinocerebral; sin embargo, además de un alto nivel de sospecha del presunto diagnóstico de mucormicosis, siempre hay que apoyarse del estudio histopatológico para el diagnóstico definitivo.

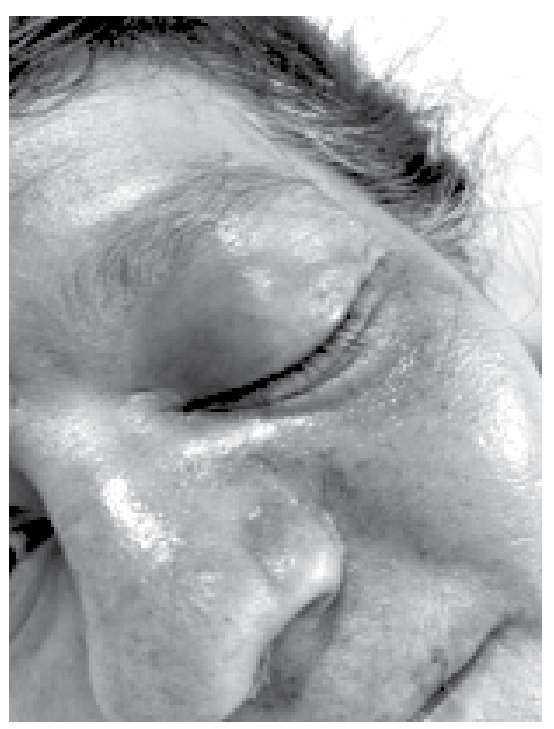

Figura 1. Se aprecia leve eritema y equimosis en el borde interno de párpado izquierdo. 


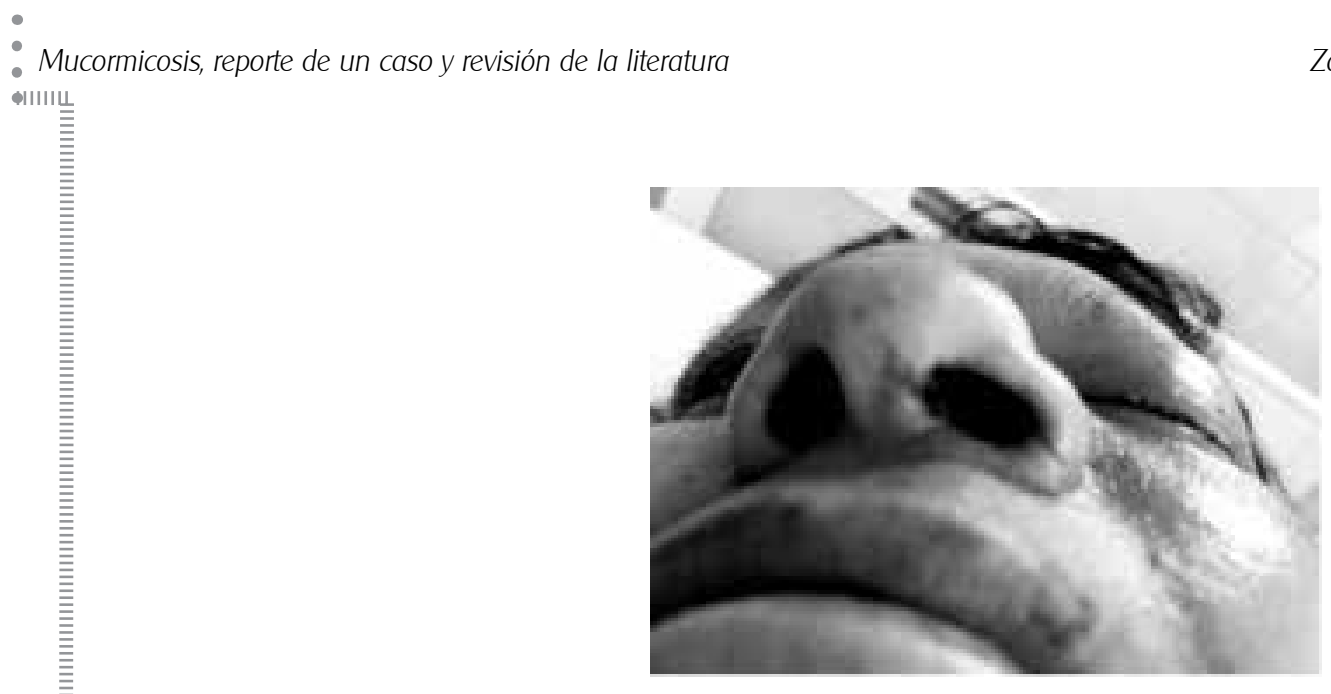

Figura 2. Se aprecia solución de continuidad lineal en la periferia de fosa nasal izquierda, así como discreta pseudo horizontalización de la fosa nasal izquierda respecto a la derecha por edena en dorso nasal.

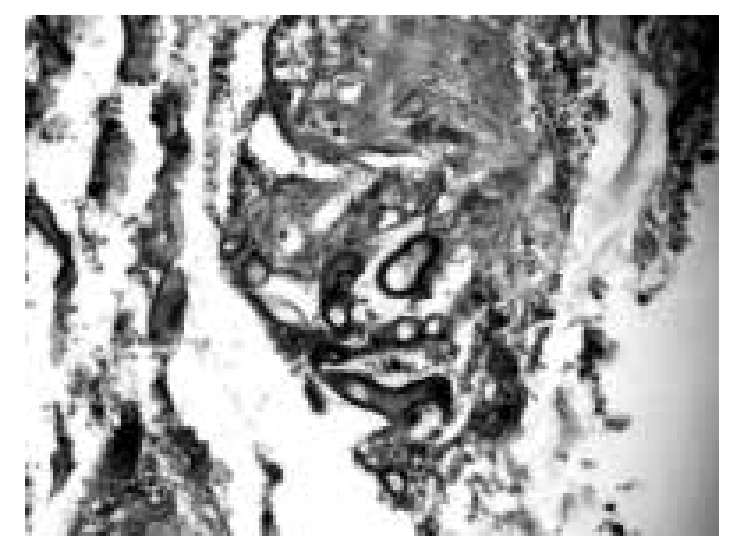

Figura 3. Corte histopatológico que evidencia filamentos sin septos y extensa destrucción tisular. Las estructuras redondas corresponden a filamentos cortados transversalmente.

\section{Bibliografía}

1. Kauffman CA, Malani AN. Zygomycosis: an emerging fungal infection with new options for management. Curr Infect Dis Rep 2007; 9(6):435-440.

2. Roden $M M$, Zaoutis TE, Buchanan $W L$, Knudsen TA, Sarkisova TA, Schaufele RL, Sein M, Sein T, Chiou CC, Chu JH, Kontoyiannis DP, Walsh TJ. Epidemiology and outcome of zygomycosis: a review of 929 reported cases. Clin Infect Dis. 2005;41(5):634-53.

3. Kontoyiannis DP, Wessel VC, Bodey GP, Rolston KV. Zygomycosis in the 1990s in a tertiary-care cancer center. Clin Infect Dis. 2000;30(6):851-856.

4. Lanternier Fanny, Sun Hsin-Yun, Ribaud Patricia, Singh Nina, Kontoyiannis Dimitrios P, Lortholary Oli-

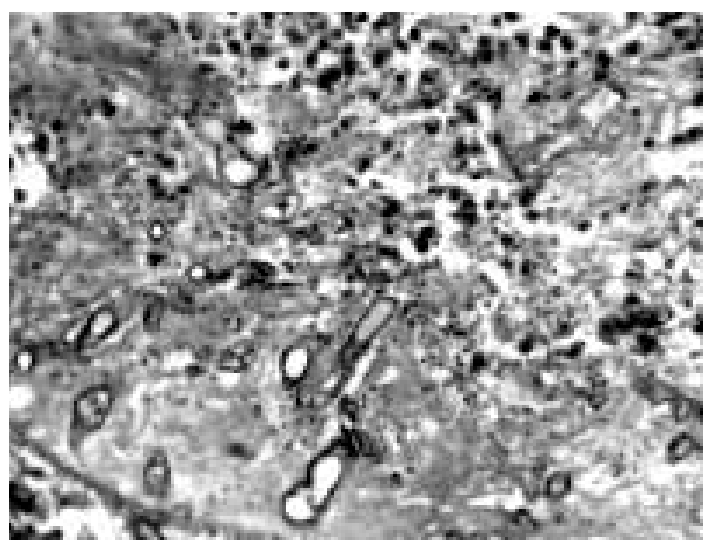

Figura 4. Corte histopatológico, que evidencia filamentos sin septos. Las estructuras redondas corresponden a filamentos cortados transversalmente. vier. Mucormycosis in organ and stem cell transplant recipients. Clin Infect Dis 2012; 54(11):1-8. doi: 10.1093/cid/cis195

5. Lelievre L, Garcia-Hermoso D, Abdoul H, Hivelin $M$, Chouaki T, Toubas D, Mamez AC, Lantieri L, Lortholary O, Lanternier F; French Mycosis Study Group. Posttraumatic mucormycosis: a nationwide study in France and review of the literature. Medicine (Baltimore). 2014 ;93(24):395-404. doi: 10.1097/ MD.0000000000000221.

6. Legrand $M$, Gits-Muselli $M$, Boutin L, Garcia-Hermoso $D$, Maurel $V$, Soussi $S$, Benyamina $M$, Ferry A, Chaussard M, Hamane S, Denis B, Touratier S, Guigue N, Fréalle $E$, Jeanne $M$, Shaal JV, Soler C, Mimoun $M$, Chaouat $M$, Lafaurie $M$, Mebazaa 
A, Bretagne S, Alanio A. Detection of Circulating Mucorales DNA in Critically III Burn Patients: Preliminary Report of a Screening Strategy for Early Diagnosis and Treatment. Clin Infect Dis. 2016 Nov 15;63(10):1312-1317.

7. Rammaert B, Lanternier F, Zahar JR, Dannaoui $E$, Bougnoux $M E$, Lecuit $M$, Lortholary $O$. Healthcare-associated mucormycosis. Clin Infect Dis. 2012 Feb;54 Suppl 1:S44-54. doi: 10.1093/cid/cir867.

8. Duffy J, Harris J, Gade L, Sehulster L, Newhouse E, O'Connell H, Noble-Wang J, Rao C, Balajee SA, Chiller T. Mucormycosis outbreak associated with hospital linens. Pediatr Infect Dis J. 2014 May;33(5):4726. doi: 10.1097/INF.0000000000000261.

9. Petrikkos G, Skiada A, Lortholary O, Roilides E, Walsh TJ, Kontoyiannis DP. Epidemiology and clinical manifestations of mucormycosis. Clin Infect Dis. 2012 Feb;54 Suppl 1:S23-34. doi: 10.1093/cid/ cir866.

10. Castrejón-Pérez Ana Daniela, Welsh Esperanza, Miranda Ivett, Ocampo-Candiani Jorge, Welsh Oliverio. Cutaneous mucormycosis. An Bras Dermatol. 2017;92(3):304प311. doi: 10.1590/abd18064841.20176614

11. Yohai RA, Bullock JD, Aziz AA, Markert RJ. Survival factors in rhino-orbital-cerebral mucormycosis. Surv Ophthalmol 1994;39(1):3-22.

12. Afroze SN, Korlepara R, Rao GW, Madala J, Contemp Clin Dent 2017;8(4):662-666.

13. Connor BA, Anderson RJ, Smith JW. Mucor mediastinitis. Chest 1979;75(4):525-526.

14. Ismail $M H$, Hodkinson HJ, Setzen G, Sofianos C,
Hole MJ Gastric mucormycosis. Trop Gastroenterol.1990;11:103-105.

15. Siddiqi SU, Freedman JD. Isolated central nervous system mucormycosis. South Med J 1994; 87:9971000.

16. Hammond SP, Bialek R, Milner DA, Petschnigg EM, Baden LR, Marty FM. Molecular methods to improve diagnosis and identification of mucormycosis. J Clin Microbiol. 2011 Jun;49(6):2151-3. doi: 10.1128/ JCM.00256-11.

17. Murphy RA, Miller WT Jr. Pulmonary mucormycosis. Semin Roentgenol 1996; 31:83-73.

18. Spellberg Brad, Walsh Thomas J, Kontoyiannis Dimitrios P, Edwards John, Ibrahim Ashraf S. Recent Advances in the Management of Mucormycosis: From Bench to BedsideClin Infect Dis. 2009 Jun 15; 48(12): 1743-1751. doi: 10.1086/599105

19. McCarthy M, Rosengart A, Schuetz AN, Kontoyiannis DP, Walsh TJ. Mold infections of the central nervous system. N Engl J Med. 2014 Jul 10;371(2):15060. doi:10.1056/NEJMra1216008

20. Chamilos G, Lewis RE, Kontoyiannis DP. Delaying amphotericin B-based frontline therapy significantly increases mortality among patients with hematologic malignancy who have zygomycosis. Clin Infect Dis. 2008 Aug 15;47(4):503-9. doi: 10.1086/590004.

21. Noxafil (posaconazole) prescribing information. https://www.merck.com/product/usa/ pi_circulars/n/noxafil/noxafil_pi.pdf (Accessed on March 18, 2014).

22. Ilharco $M$, Pereira $C M$, Moreira $L$, Procenca $A L$, Fevereiro MC, Lampreia F, Rola J. Rhinoorbital Mucormycosis in the immunocompetent: Experience with Isavuconazole ,ID Cases 2019 Jul 9;18: e00591. 